\title{
First Report of Sour Rot on Post-harvest Oriental Melon, Tomato, Cucumber, Potato, Pumpkin and Carrot Caused by Geotrichum candidum
}

\author{
Yong-Ki Kim*, Taek-Soo Kim¹, Hong-Sik Shim², Kyung-Seok Park', Wan-Hae Yeh², Sung-Jun Hong, \\ Chang-Ki Shim, Jeong-Soon Kim, Jong-Ho Park, Eun-Jung Han, Min-Ho Lee and Hyeong-Jin Jee \\ Organic Agriculture Division, National Academy of Agricultural Science (NAAS), RDA, Suwon 441-707, Korea \\ ${ }^{1}$ Agricultural Microbiology Team, NAAS, RDA, Suwon 441-707, Korea \\ ${ }^{2}$ Crop Protection Division, NAAS, RDA, Suwon 441-707, Korea
}

(Received on June 16, 2011; Revised on August 6, 2011; Accepted on August 6, 2011)

\begin{abstract}
During survey of postharvest diseases of vegetables in the middle region of Korea in 2003, 2004 and 2005, new disease symptoms showing watery rot and soft rot were observed. In this study, the disease causal agents were identified as Geotrichum candidum, and their host range and pathogenicity were investigated. G. candidum isolated had wide host range and strong pathogenicity against carrot, cucumber, tomato and pumpkin. The disease occurrence on several vegetables that $G$ candidum can be a serious threat to stable production of fresh vegetable.
\end{abstract}

Keywords : Geotrichum candidum, Postharvest, Sour rot

During survey of postharvest diseases of vegetables in the middle region of Korea in 2003, 2004 and 2005, new disease symptoms showing watery rot and soft rot were observed. The disease symptoms were found on oriental melon, tomato, radish, potato and carrot. The disease casual agents were isolated and inoculated to confirm their pathogenicity. Initial symptoms were appeared as water-soaked lesions and white mycelia formation on infected parts. When plant tissues were affected, out layers of rind were intact but inner parts were decayed completely. And then out layer of rind were cracked. The isolated casual agents were identified based on cultural, mycological and bio-chemical characteristics by comparing with those of two representative type cultures, Geotrichum candidum KACC41323 and Geotrichum species KACC40367 given from Korean Agriculture Culture Collection (KACC). The cultural, mycological and bio-chemical characteristics of the present isolates were very similar with those of Geotrichum species (Table 1). The present isolates formed hyaline, smooth, one-celled, subglobose to cylindrical arthroconidia. The arthroconidia were quite variable in size. On trptic soy agar, colonies were fast growing, flat, white to cream, dry and finely suede-like with no reverse pigment. Hyphae were hyaline, septate,

*Corresponding author

Phone) +82-31-290-0554, Fax)+82-31-290-0507

Email)yongki@rda.go.kr branched and break up into chains of hyaline, smooth, one-celled, subglobose to cylindrical arthroconidia (Fig. 1). Odor like it from apple was strongly given off on the present isolates-cultured agar.

In order to identify precisely the isolated yeast at species level, utility of carbon and nitrogen sources were investigated using Biolog YT microplate. As a result, all the isolates tested were identified as Geotrichum candidum with similarity at the range of 42 to $76 \%$ (Table 2). Finally we identified the six isolated yeasts as G. candidm. In order to confirm host range and pathogenicity of the six newly-identified $G$. candidum isolates, ten vegetable crops including potato, sweet potato, carrot, melon and oriental melon were inoculated with conidial suspension of each G. candidum isolates. The present G. candidum isolates caused typical symptoms on the challenged vegetables (Fig. 2).

Pathogenicity of $G$ candidum tested showed high differences among the isolates. G. candidum OM-1 isolate from oriental melon showed high pathogenicity against tomato, cherry tomato and pumpkin, but no pathogenicity against oriental melon. To-1 isolate from tomato showed a little pathogenicity to tomato, cherry tomato and oriental melon. HY-1 isolate from radish showed pathogenicity to cucumber, oriental melon, tomato and cherry tomato. HY-5 isolate from potato had wide host range and showed pathogenicity to potato, carrot, cucumber, tomato, cherry tomato and pumpkin. B-34 isolate from carrot showed pathogenicity 
Table 1. Cultural, mycological and biochemical characteristics of the two representative isolates Geotrichum sp. and G. candidum, and the present isolates obtained from postharvested vegetables in Korea

\begin{tabular}{|c|c|c|c|c|c|c|c|}
\hline \multirow{2}{*}{ Characteristics } & \multicolumn{2}{|c|}{ Representative isolates $^{\mathrm{a}}$} & \multicolumn{5}{|c|}{ Present isolates $^{\mathrm{b}}$} \\
\hline & Gs & Gc & OM-1 & TO-1 & HY-1 & HY-5 & B-34 \\
\hline Intense smell (Pineapple) & + & + & + & + & + & + & + \\
\hline Rapidly growing & + & + & + & + & + & + & + \\
\hline White and dry mycelium & + & + & + & + & + & + & + \\
\hline Powdery to cottony colonies & + & + & + & + & + & + & + \\
\hline Not grow at 37 & - & - & - & - & - & - & - \\
\hline Spore size $(\mu \mathrm{m})$ & $6.5 / 2.9$ & $5.6 / 2.2$ & $6.2 / 3.1$ & $6.2 / 2.7$ & $6.3 / 3.1$ & $6.3 / 3.5$ & $6.5 / 3.0$ \\
\hline Mycelium width $(\mu \mathrm{m})$ & 6.7 & 7.6 & 7.1 & 6.7 & 7.5 & 8.0 & 6.6 \\
\hline Optimal Growth temperature $\left({ }^{\circ} \mathrm{C}\right)$ & $25-28$ & $25-28$ & $25-28$ & $25-28$ & $25-28$ & $25-28$ & $25-28$ \\
\hline \multicolumn{8}{|c|}{ Growth on sole carbon source $(1 \%, w / v)$} \\
\hline Glucose & - & + & + & + & + & + & + \\
\hline D-Galactose & - & + & + & + & + & + & + \\
\hline Glycerol & - & + & + & + & + & + & + \\
\hline D-manitol & - & + & + & + & + & + & + \\
\hline
\end{tabular}

${ }^{\mathrm{a}} \mathrm{Gs}$, Geotrichum sp.; Gc, Geotrichum candidum.

${ }^{\mathrm{b}} \mathrm{OM}-1$, TO-1, HY-1, HY-5, and B-34 were isolated from harvested oriental melon, tomato, radish, potato and carrot, respectively.

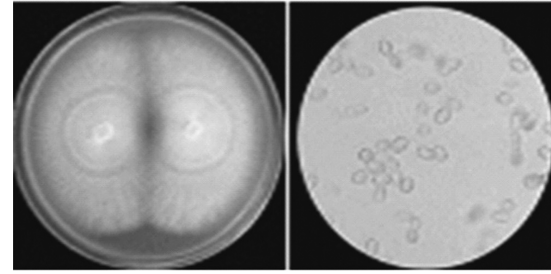

Geotrichum candidum

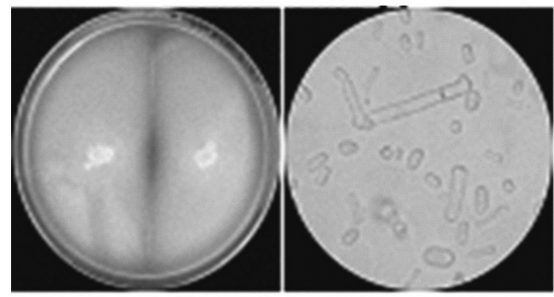

Geotrichumsp.

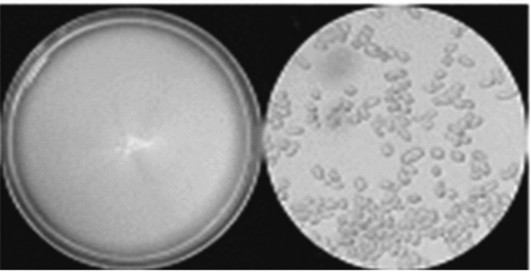

TO-1(OM-1, HY-1, HY-5)

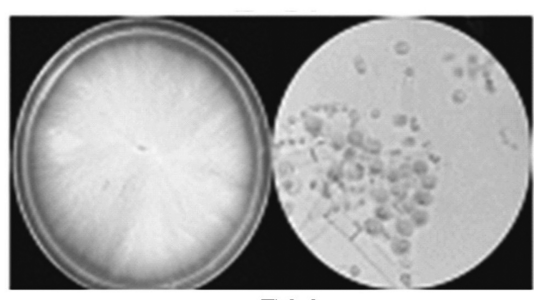

B34

Fig. 1. Morphological characteristics of the two representative isolates, G. candidum and Geotrichum species and the present isolates of Geotrichum obtained from harvested vegetables. Left and right plates of each figure represent colony pattern and arthroconidia formed on tryptic soy agar after five day-incubation.

Table 2. Identification, similarity index and nearest species for six yeast isolates obtained from harvested vegetables according to Biolog microstation (YT microplate) for yeast identification

\begin{tabular}{lcccl}
\hline \hline Isolates & Host & Identification & $\begin{array}{c}\text { Similarity } \\
(\%)\end{array}$ & Closest species \\
\hline OM-1 & Cucumber & G. candidum & 62 & Candida sorbophila \\
To-1 & Tomato & $"$ & 70 & C. sorbophila \\
HY-1 & Radish & $"$ & 76 & C. rugosa B \\
HY-5 & Potato & $"$ & 76 & C. sorbophila \\
B-34 & Carrot & $"$ & 99 & C. sorbophila \\
\hline
\end{tabular}

to carrot, cucumber, oriental melon and pumpkin. Consequently there was big difference in host range and pathogenicity among $G$ candidum isolates from different vegetable crops (Table 3).

In this study, isolates OM-1 and HY-1 isolated from harvested oriental melon and radish were not pathogenic to oriental melon and radish in pathogenicity test of seven days, respectively. The reason is not clear and I think, further study for its elucidation will be needed.

Until now, few reports on sour rot caused by $G$. candidum has been reported only on citrus fruit in 


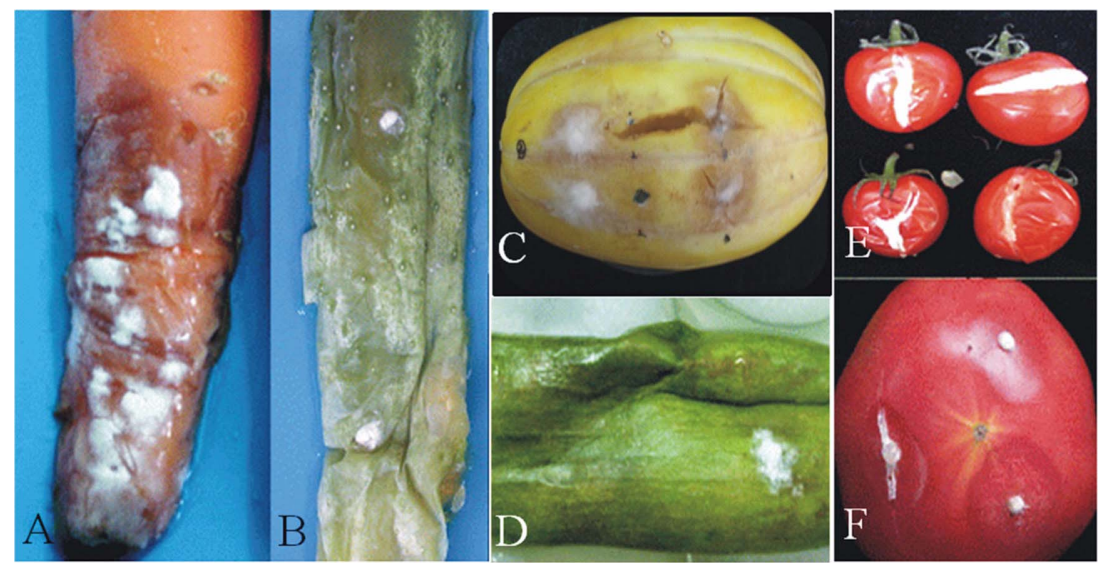

Fig. 2. Typical symptoms of watery rot formed on carrot, cucumber, pumpkin, oriental melon, cherry tomato and tomato when they were inoculated with Geotrichum candidum isolate B34 (A and D), isolate OM-1 (B and C) and isolate To-1 (E and F). B-34, OM-1 and TO-1 were isolated from harvested carrot, oriental melon and tomato, respectively.

Table 3. Pathogenicity of Geotrichum candidum isolated from stored vegetables and potato

\begin{tabular}{|c|c|c|c|c|c|c|c|}
\hline \multirow[t]{2}{*}{ Crop } & \multicolumn{7}{|c|}{$\begin{array}{l}\text { Pathogenicity }{ }^{\mathrm{a}} \text { of Geotrichum candidum } \\
\text { isolated from different crops }\end{array}$} \\
\hline & Gs & Gc & OM-1 ${ }^{\text {b) }}$ & To-1 & HY-1 & HY-5 & B-34 \\
\hline Potato & \pm & - & - & - & - & + & \pm \\
\hline Sweet potato & \pm & - & \pm & \pm & - & - & - \\
\hline Carrot & +++ & + & - & + & - & +++ & ++ \\
\hline Melon & - & + & \pm & \pm & \pm & \pm & - \\
\hline Radish & - & \pm & \pm & \pm & - & - & - \\
\hline Cucumber & +++ & ++ & - & - & ++ & ++ & +++ \\
\hline Oriental melon & + & - & - & + & ++ & - & + \\
\hline Tomato & +++ & - & +++ & + & +++ & + & - \\
\hline Cherry tomato & +++ & - & +++ & + & +++ & + & - \\
\hline Pumpkin & ++ & + & +++ & - & - & + & +++ \\
\hline
\end{tabular}

${ }^{\mathrm{a}}-, \pm,+,++,+++$ represents no, very little, little, moderate and strong pathogenic, respectively. Pathogenicity of each isolates was evaluated three days after inoculation of each isolate.

${ }^{\mathrm{b}} \mathrm{OM}-1, \mathrm{TO}-1, \mathrm{HY}-1, \mathrm{HY}-5$ and B-34 were isolated from harvested oriental melon, tomato, radish, potato and carrot, respectively.

Korea (Park, 1961; Song, 1997; Hyun, 2002). However, it has been known to have ability to infect several postharvest plants, such as citrus fruit (Suprapta et al., 1995; Butler et al., 1988), peach (Crisosto et al., 2004; Wells, 1977), tomato (Oladiran et al., 1993), apple, pear, egg plant, carrot, cucumber, guava, melon, papaya, pumpkin, sponge gourd and turnip (Fatima et al., 2009; Saude et al., 2005) throughout whole countries.

This is first report of sour rot on vegetables like oriental melon, tomato, cherry tomato, cucumber, potato, pumpkin and carrot caused by G. candidum. G. candidum isolated had wide host range and strong pathogenicity against carrot, cucumber, tomato and pumpkin. The disease occurrence on several vegetables suggests that
G. candidum can be a serious threat to stable production of fresh vegetable.

\section{Acknowledgement}

This study was carried out with the support of "Research Program for Agricultural Science \& Technology Development (Project No. PJ0068872011)", National Academy of Agricultural Science, Rural Development Administration, Republic of Korea.

\section{References}

Butler, E., Fogle, D. and Miranda, M. 1988. Galactomyces citriaurantii a newly found teleomorph of Geotrichum citri-aurantii : Cause of sour rot of citrus fruit. Mycotaxon 33: 197-212.

Fatima, N., Batool, H., Sultana, V., Ara, J. and EhteshamulHaque, S. 2009. Prevalence of post-harvest rot of vegetables and fruits in Karachi, Pakistan. Pak. J. Bot., 41: 3185-1390.

Hyun, J. W. 2002. Compendium of citrus diseases and pests. Citrus Research Station, National Institute of Horticultural and Herbal Science, Republic of Korea.

Oladiran, A. O. and Iwu, L. N. 1993. Studies on the fungi associated with tomato fruit rots and effects of environment on storage. Mycopathologia 121: 157-161.

Park, J. S. 1961. Research Report 2:19. Agricultural college of Chungnam National University. (in Korean)

Saude, C. and Hausbeck, M. K. 2005. Sour rot. in Black rot of carrots.

Song, J. H. 1997. Current status of the occurrence of citrus diseases and etiological studies on citrus scab, sclerotia twig blight and cottony rot and sour rot. M. Sc. thesis, University of Sunchon Nation University, Republic of Korea.

Suprapta, D. N., Arai, K. and Iwai, H. 1995. Distribution of Geotrichum candidum citrus race in citrus groves and noncitrus field in Japan. Mycoscience 36: 277-282.

Wells, J. M. 1977. Sour rot of peaches caused by Monilinia implicate and Geotrichum candidum. Phytopathology 67: 404-408. 